\title{
REFORMAS INSTITUCIONALES E INTEGRACIÓN GLOBAL LAINTERVENCIÓN DEL BANCO MUNDIAL EN ARGENTINA DURANTE LOS '90'
}

\author{
Ruth Felder
}

\begin{abstract}
RESUMO
El proceso de reformas económicas e institucionales llevado a cabo en Argentina durante la década de los '90 transformó profundamente la estructura económica del país, las formas de acción del estado y la configuración de su aparato institucional. En este trabajo revisaré la intervención del Banco Mundial en las distintas etapas de este proceso. Prestaré atención al rol que éste tuvo en la primera etapa de reducción del tamaño del aparato del Estado y de apertura al mercado. Luego analizaré los argumentos y proyectos referidos al fortalecimiento de las capacidades institucionales del Estado y a la creación de redes de contención social desarrollados en el marco de la denominada 'Segunda Reforma del Estado' de mediados de los '90. Finalmente, me centraré en el abandono de estas prioridades relacionadas con fortalecimiento del Estado y en la creciente importancia que adquirieron los proyectos relacionados con el ajuste fiscal y el manejo de la deuda pública en el contexto de los desequilibrios macroeconómicos y de la recesión de fines de los '90. A partir del análisis de la dinámica histórica de la intervención del Banco Mundial en el proceso de reformas, voy a plantear que, más allá de los objetivos formales de los numerosos proyectos del Banco Mundial, la institución ha jugado un rol mediador entre los requerimientos de integración en la economía global y el Estado argentino tanto en la etapa de 'reducción' como en la de 'mejoramiento' de la intervención del Estado. Sus proyectos se han orientado a transformar estos requerimientos en esquemas institucionales y en políticas estatales concretos que profundizaron la integración del país en la economía global. Estas prioridades se han puesto de manifiesto en la crisis de fines de los '90 y principios de la presente década.
\end{abstract}

PALAVRAS-CHAVE: Argentina; Banco Mundial; reforma del Estado; neoliberalismo.

\section{INTRODUÇÃO}

En 1989, en Argentina se inició un proceso de reformas económicas e institucionales inspiradas en las premisas neoliberales de privatización de activos públicos, desregulación de los mercados (incluido el mercado de trabajo), apertura comercial y financiera y disciplina fiscal y monetaria. Estas reformas no sólo modificaron las funciones y la configuración del aparato estatal, sino que también implicaron una transformación de la estructura económica y de las relaciones sociales dentro del país y de los vínculos entre la economía local y la internacional. Esta transformación

\footnotetext{
1 Agradezco los comentarios de Leo Panitch y de Viviana Patroni a una versión mucho más extensa de este trabajo y las observaciones de Maria Antonieta Leopoldi a un borrador del artículo. Como es usual, asumo toda la responsabilidad por lo que se dice aquí.
}

culminó en 2001 en una crisis económica, política y social de alcances inéditos.

La activa intervención del Banco Mundial expresada en el financiamiento de numerosos proyectos de ajuste y reforma institucional, en el diálogo permanente con las autoridades argentinas acerca de las políticas en curso y en la elaboración de diagnósticos y análisis acerca de la evolución de la economía y de la situación del país - fue un rasgo saliente del proceso de reformas. Esta intervención ha sido fuertemente criticada por quienes responsabilizaron al Banco Mundial (y al Fondo Monetario Internacional - FMI) por la crisis que sufrió la Argentina hacia finales de la década del ' 90 y defendida por quienes han atribuido la crisis a la falta de decisión política de los gobiernos argentinos para completar las reformas recomendadas por la institución. En la mayoría de los casos, estos argumentos se han apoyado en visiones generales acerca del sentido de la acción del Ban-

Rev. Sociol. Polít., Curitiba, v. 17, n. 33, p. 55-68, jun. 2009 
co Mundial, más que análisis sistemáticos de la misma y de la dinámica histórica de la relación entre el Banco y el Estado argentino.

Este trabajo se propone revisar las modalidades de intervención del Banco Mundial en este proceso. Para hacerlo, parte de la premisa de que la reforma del Estado es un proceso político y, como tal, conflictivo. Más claramente, las políticas reformistas no son la mera implementación de esquemas técnicos tendientes a lograr configuraciones institucionales, mecanismos de gestión y asignación de recursos óptimos, sino que constituyen una compleja dinámica histórica en la que el Estado ha respondido de maneras particulares y cambiantes a las demandas de distintos actores sociales, en escenarios económicos y políticos nacionales e internacionales también cambiantes. La intervención del Banco Mundial debe situarse en este contexto histórico de transformación de las condiciones económicas nacionales e internacionales y de las cambiantes relaciones de fuerza entre los sujetos directa o indirectamente involucrados en el desarrollo de las reformas. Vista de este modo, esta intervención no es un programa técnico coherente que fue incorrectamente aplicado, como han argumentado sus defensores. Tampoco es, como han planteado muchos críticos, la imposición externa de un diagnóstico y una estrategia de reformas erróneos o malintencionados que desembocaron en la profunda crisis de 2001. Es, en cambio, una forma de mediación a través de la cual cambiantes condiciones económicas nacionales e internacionales y nuevas relaciones de fuerzas favorables al capital se 'tradujeron' en esquemas institucionales y en políticas estatales, que a su vez catalizaron procesos de transformación estructural de la economía y la sociedad argentina. Las aparentes contradicciones de la estrategia reformista y los resultados decepcionantes en muchas áreas de intervención estatal no deben opacar el hecho de que se produjo una transformación general del rol del Estado argentino y de las relaciones de fuerzas de las que éste es parte.

En lo que sigue, revisaré brevemente el escenario de crisis de fines de los ' 80 , en el que se legitimó la necesidad de llevar adelante una reforma estructural del Estado. Luego, reseñaré la etapa inicial de esta reforma, en la que se redujo el tamaño del aparato del Estado y se crearon oportunidades para el ingreso de capitales privados en áreas que previamente eran responsabilidad estatal exclusiva. Me centraré en algunos resultados críticos de esta primera etapa que, a partir de mediados de los '90, llevaron a que se planteara la necesidad de una 'segunda reforma del Estado' en la que se establecieron nuevos objetivos y prioridades para la acción estatal en un marco de revalorización teórica del rol del Estado. Identificaré las novedades introducidas y reflexionaré acerca de las razones que hicieron que estas reformas no cumplieran con sus fines explícitos de lograr un desarrollo sustentable, garantizando la protección de los grupos sociales negativamente afectados por las transformaciones económicas. Finalmente, me ocuparé del cambio de prioridades de las políticas reformistas en el marco de la recesión y de las dificultades financieras de fines de la década del '90. En cada una de estas etapas, prestaré especial atención a los argumentos planteados por el Banco Mundial y a los proyectos a través de los cuales se ha materializado su intervención.

\section{LA VÍSPERA DE LAS REFORMAS}

Para entender el proceso de reformas de los '90, es necesario remontarse a la crisis hiperinflacionaria de fines de los ' 80 y a las condiciones de gestación de esta crisis durante la dictadura militar que tomó el poder en 1976. La dictadura tuvo el propósito explícito de desarticular la estrategia de desarrollo por sustitución de importaciones vigente hasta entonces y las formas de intervención estatal, articulación social e inserción internacional de la economía argentina que la habían acompañado. Para cumplir con este propósito, se llevaron adelante políticas económicas inspiradas en la nueva ortodoxia de mediados de los '70 que prescribía la disciplina monetaria y fiscal, el estímulo a las exportaciones y al ingreso de capitales y la recuperación de las tasas de ganancia capitalistas mediante la reducción de los costos laborales.

En un contexto global de liquidez monetaria, se eliminaron los obstáculos al movimiento de capitales y se elevaron las tasas de interés locales, convirtiendo a la Argentina en un destino atractivo para inversores financieros. Al revertirse esta situación de afluencia de capitales y, muy especialmente, luego de la crisis de la deuda externa mexicana de 1982, el Estado argentino estableció diversos mecanismos tendientes a obtener divisas, cumplir con los compromisos derivados de la deuda externa y hacer frente a las amenazas de creciente inestabilidad financiera. Estas políticas estatales culminaron con 
la estatización de la deuda externa privada en 1982 o, lo que es lo mismo, con la socialización de los costos de la estrategia de valorización de quienes se habían endeudado en mercados financieros internacionales e invertido en el mercado local aprovechando las altas tasas de interés.

Luego de asumir la deuda externa privada, el estado encaró la renegociación de sus condiciones. Los bancos acreedores demandaban un acuerdo previo con el FMI para iniciar las negociaciones. Éste, por su parte, exigía un ajuste económico al que el gobierno accedió. Luego, la creciente oposición social y política a la dictadura impidió la concreción de este ajuste, agudizó la incertidumbre económica, llevó a la interrupción de las negociaciones con los acreedores privados y el FMI y a la convocatoria a elecciones presidenciales. ${ }^{2}$ Aunque la política económica de la dictadura no logró sus objetivos explícitos (entre ellos, controlar la inflación y mejorar la competitividad de la economía argentina), en este período se produjeron importantes reacomodamientos de precios relativos a favor de los productores de bienes no transables y en detrimento de los asalariados y sectores de la economía expuestos a la competencia externa. La deuda asumida por el Estado constituiría un condicionante que desde entonces limitaría sus márgenes de acción y sería una de las claves de la crisis de fines de los '80 y de las razones que legitimarían las reformas de los ' 90 .

La recuperación democrática de 1983 y la gestión del primer presidente electo, Raúl Alfonsín, estuvieron signadas por los desequilibrios heredados de la dictadura combinados con la multiplicación de las demandas sociales previamente reprimidas por el régimen autoritario. Luego de un primer intento de apelar a la buena voluntad de los acreedores para reducir el peso del endeudamiento, en 1984 el gobierno argentino aceptó la intervención del FMI, la cual se tradujo en compromisos de ajuste fiscal y externo. A partir de 1987, frente a la persistencia de los desequilibrios macroeconómicos, comenzaron a definirse diversos proyectos de reforma estructural del Estado y la economía. En este marco, el Banco Mundial otorgó un préstamo para el ajuste estructural (Structural Adjustment Lending - SAL)

$2 \mathrm{Al}$ descontento que generaban los efectos negativos de las políticas económicas, en 1982 se le sumó la derrota militar a manos de Gran Bretaña en la guerra de Malvinas. que ofrecía desembolsos rápidos tendientes a mejorar la balanza de pagos a cambio del compromiso de liberalizar el comercio exterior y los mercados financieros, reestructurar las empresas públicas, mejorar la competitividad de la agricultura y la industria y reducir el gasto públiCo (TORRE, 1997). Este acuerdo entre el gobierno argentino y el Banco Mundial expresó un desplazamiento en las concepciones acerca de los problemas macroeconómicos del país, que pasaron de ser entendidos como desajustes de corto plazo, producto de la mala gestión de la dictadura, a interpretarse como resultado de desequilibrios estructurales. En este viraje, el Banco Mundial asumió el liderazgo de las reformas, desplazando de este rol al FMI (SCHVARTZER, 1997; MOSLEY, HARRIGAN \& TOYE, 1991) ${ }^{3}$.

El gobierno argentino enfrentó creciente oposición política para implementar los ajustes y las reformas acordados. A pesar de que el Estado argentino no cumplió con las condiciones del acuerdo, el Banco Mundial mantuvo su apoyo y siguió proveyendo fondos para acelerar la reestructuración económica hasta 1988. En ese año, la Argentina suspendió el pago de los intereses de la deuda pública con los bancos internacionales (TEICHMAN, 1997). Luego de que fracasaran las negociaciones con el FMI y con los acreedores privados tendientes a reanudar los pagos, el Banco Mundial interrumpió sus desembolsos. La interrupción de las negociaciones con el FMI y los acreedores privados profundizó la inestabilidad macroeconómica y contribuyó a que se generaran las condiciones para una corrida especulativa contra la moneda, que desembocó en un pico hiperinflacionario, una aguda crisis económica y social y en la asunción anticipada de la presidencia por parte de Carlos Menem en julio de 1989.

A la luz de este fracaso, la acción del Banco Mundial fue objeto de críticas. Por una parte, la institución fue responsabilizada por conceder préstamos a un país insolvente (MOSLEY, HARRIGAN \& TOYE, 1991). Por otra, el

\footnotetext{
3 De acuerdo con la división de tareas entre las instituciones financieras internacionales establecida en el momento de la creación de ambas en el marco de los acuerdos de Bretton Woods, el Banco Mundial tiene a su cargo tareas relacionadas con las estrategias de desarrollo de largo plazo mientras que el Fondo Monetario Internacional se encarga del manejo de las variables macroeconómicas y de responder a desequilibrios macroeconómicos.
} 
préstamo del Banco no había estado precedido por un programa de ajuste del FMI como era usual. Más aún, la institución había extendido su intervención al manejo de desequilibrios macroeconómicos, lo que se consideraba atribución del FMI y, al hacerlo, había relajado las condicionalidades estrictas que se asocian a la asistencia financiera del Fondo (TUSSIE \& BOTZMAN, 1990). Luego de los cuestionamientos por parte de EUA y de otros países centrales, ambas instituciones establecieron un concordato por el cual en lo sucesivo se comprometían a resolver sus diferencias lo más rápidamente posible y a evitar hacer recomendaciones contradictorias a los países (BURNHAM, 1999). Desde entonces, el Banco Mundial sólo otorgaría préstamos a países que hubieran formalizado previamente un acuerdo con el FMI.

Si bien el programa de ajuste estructural de los '80 no logró equilibrar la economía, tuvo otros resultados que serían determinantes en los años siguientes: aumentó la influencia del Banco en el país y contribuyó a instalar la reforma del estado y el ajuste estructural como un tema prioritario de la agenda pública. En este sentido, cabe citar un documento del Banco en el que se justificaba la continuidad de la asistencia al país, explicando que la institución había asumido un 'riesgo calculado' que había permitido al país “comprar tiempo para mantener su calendario electoral intacto, mientras construía el apoyo social para las reformas estructurales, evitaba el default con el Banco, posponiendo la acumulación de atrasos con el Fondo y dando los primeros pasos de la reforma impositiva que luego beneficiarían al nuevo gobierno" (BANCO MUNDIAL, 1996a, p. 24).

\section{LA ETAPA 'VIRTUOSA’ DE LAS REFOR- MAS ESTRUCTURALES}

Carlos Menem asumió la Presidencia de la Argentina en 1989, comprometiéndose a llevar adelante las reformas para resolver definitivamente la crisis. Atribuyendo la hiperinflación y el estancamiento económico al tamaño exagerado del aparato del Estado, al gasto público desmedido y a la intervención económica estatal ineficiente y excesiva, el nuevo Presidente anunció su intención de reducir el aparato del Estado, limitar el gasto público, eliminar definitivamente el problema de la deuda pública, restringir la intervención estatal en la economía y crear oportunidades atractivas para inversores privados y para el desarrollo de los mercados. En menos de cinco años, la mayoría de las empresas públicas fueron privatizadas, se modificó la estructura de la administración pública, se redujo el número de empleados públicos, se transfirieron servicios a las administraciones provinciales y se redefinieron las relaciones fiscales entre la administración nacional y las provincias, se privatizó parcialmente el sistema de seguridad social, se desreguló un conjunto importante de actividades económicas, se eliminaron diversos impuestos, se liberalizó el mercado de capitales y se flexibilizaron las relaciones laborales. La demostrada voluntad política de llevar adelante reformas estructurales le permitió al gobierno atraer masivos flujos de capitales durante los primeros años de la década del '90 y ganar el apoyo de las instituciones financieras internacionales.

Al momento de la asunción de Carlos Menem, el Banco Mundial había interrumpido la transferencia de fondos hasta tanto "se lograran avances en administración macroeconómica y reducción de la deuda con bancos comerciales" (BANCO MUNDIAL, 1990) ${ }^{4}$. Sin embargo, la institución participó activamente en la formulación de la estrategia inicial de reformas a través de estudios, asesoramiento y diálogo con el gobierno argentino. También elaboró un diagnóstico comprehensivo que guiaría su intervención durante los años siguientes. En este diagnóstico se señalaba a la estrategia de crecimiento estatista y cerrada y a la existencia de 'grupos de interés' privilegiados como las causas de los desequilibrios macroeconómicos (BANCO MUNDIAL, 1996a y 1996b $)^{5}$. Acompañando este diagnóstico, la

\footnotetext{
4 Country Strategy Paper de 1990, citado en BANCO MUNDIAL (1996a, p. 66).

5 Entre los grupos de interés detectados por el Banco Mundial se cuentan los trabajadores sindicalizados que contaban con protecciones y derechos laborales, la industria local, quienes habían accedido a la vivienda gracias a subsidios estatales, los productores agrícolas de productos cuyos precios estaban regulados, los consumidores que se beneficiaban con servicios públicos baratos etc. (BANCO MUNDIAL, 1996b). La descripción es tan exhaustiva que podría parecer absurda al menos que se la interprete como un cuestionamiento radical a las modalidades de distribución de recursos por fuera de los mecanismos de mercado. La noción de grupos de interés o intereses sectoriales resulta aquí una forma de descalificar cualquier alternativa al libre mercado, libre comercio y libre movimiento de capitales (acerca de la descalificación de cualquier argumento que se aparta del libre mercado, ver WADE, 2001).
} 
institución apoyó el plan de reformas y este apoyo se reflejó rápidamente en el otorgamiento de créditos que financiaron la privatización de diversas empresas públicas y en la activa participación de técnicos del Banco en la definición e implementación de la política privatizadora.

A estas primeras reformas les siguió la implementación del Plan de Convertibilidad en 1991. Este plan, cuyo propósito explícito era reducir la persistente inflación, estableció una tasa de cambio fija entre el peso argentino y el dólar y determinó que el Banco Central debía mantener un monto de reservas de divisas equivalentes al monto de dinero circulante en la economía, como respaldo al valor de la moneda local. Cualquier oscilación en este monto de reservas debía reflejarse en el monto de dinero circulante. Por otra parte, el Banco Central se obligaba a cambiar dólares y pesos sin restricciones. $\mathrm{La}$ implementación de la Convertibilidad permitió reducir la inflación de manera rápida y drástica y redujo el rol del Banco Central al de una caja de conversión.

El inicio de las reformas estructurales y la estabilización macroeconómica hicieron posible una renegociación de la deuda pública por la cual la deuda de corto plazo se transformó en bonos de largo plazo, siguiendo los lineamientos del Plan Brady para la renegociación de las deudas latinoamericanas. Esta renegociación se formalizó en 1993 mediante un acuerdo con el FMI y los bancos comerciales y un préstamo el Banco Mundial (BONNET, 2003; GAMBINA, 2003; KULFAS \& SCHORR, 2003). La adhesión al Plan Brady permitió reducir los intereses que el Estado argentino tendría que pagar en los años siguientes, a cambio de un compromiso de no incurrir en atrasos en los pagos y de mantener el superávit fiscal. De este modo, el país recobró su acceso a los mercados financieros internacionales, lo que, sumado a la creación de nuevas oportunidades de negocios a través de la privatización de activos públicos, la estabilización, las altas tasas de interés locales y un proceso de apertura financiera, alentó el ingreso masivo de capitales, dio lugar a una recuperación económica y contribuyó a recrear la confianza social en la perspectiva de un crecimiento sostenido basado en las reformas estructurales (BASUALDO, 1999).

Las expectativas optimistas comenzarían a frustrarse cuando, hacia mediados de la década, los capitales que pocos años antes se habían volcado a los llamados 'mercados emergentes' (entre los cuales se incluía a la Argentina) se reorientaron hacia los países centrales. Para la misma época, comenzaron a hacerse visibles un conjunto de dificultades resultantes de las propias reformas ${ }^{6}$. En este contexto, el Estado comenzó a aumentar aceleradamente su endeudamiento, a efectos de mantener las reservas de divisas según lo dispuesto por el programa de convertibilidad y de paliar el déficit fiscal. El deterioro de la situación económica y las crecientes dificultades fiscales en un marco de ausencia de restricciones a los movimientos de capitales hicieron que las sucesivas crisis financieras y la volatilidad financiera mundial de la segunda mitad de los '90 golpearan particularmente al Estado argentino, que veía encarecerse el crédito y carecía de instrumentos para limitar la fuga de capital ${ }^{7}$. Paralelamente, tasas crecientes de desempleo y empobrecimiento, sumadas a los menguados recursos estatales para asistir a los afectados por el ajuste económico dieron lugar a un recrudecimiento del conflicto social, el cual había sido menos visible en la primera etapa de estabilización macroeconómica.

Como respuesta a estas tensiones, se planteó la necesidad de llevar adelante reformas que fortalecieran las capacidades institucionales del estado mientras se mantenía el gasto público bajo control. Así, se podría retener la confianza de los inversores, limitar los efectos disruptivos de los mercados desregulados y aliviar las situaciones sociales más críticas. En esta tónica, en 1996, el gobierno anunció el inicio de la denominada 'segunda reforma del Estado' que se proponía profundizar los procesos de apertura y desregulación económica y mejorar la capacidad de las instituciones estatales, con el objetivo formal de potenciar el crecimiento económico y favorecer el desarrollo y la equidad social en una sociedad regida por la lógica del mercado.

\footnotetext{
6 Fundamentalmente, la pérdida de competitividad de la economía como resultado de la apertura comercial y la sobrevaluación de la moneda, el incremento de la desocupación y la caída de los salarios reales.

${ }^{7}$ Cabe recordar que se habían eliminado las restricciones a los movimientos de capital y que, de acuerdo con el Plan de Convertibilidad, el Banco Central estaba obligado a entregar al público todos los dólares que le fueran solicitados.
} 


\section{DE MENOR A MEJOR: EL REDESCUBRI- MIENTO DEL ESTADO EN EL MARCO DE LAS REFORMAS}

La revalorización del rol del Estado que se produjo en la Argentina en la segunda mitad de la década del '90 se inscribió en el marco de un debate intelectual internacional en el que se destacaba la importancia del Estado como promotor del desarrollo y como sustento legal e institucional necesario para el funcionamiento de los mercados. A partir de estas ideas se fue consolidando un nuevo consenso acerca de la necesidad de prestar atención a los aspectos políticos y sociales de las reformas y a las dimensiones institucionales del funcionamiento económico (ROXBOROUGH, 1997). Muy sintéticamente, el argumento central es que las deficiencias de la intervención estatal (v.g. arbitrariedad, impredictibilidad, falta de cumplimiento de los contratos etc.) son las principales razones de la escasez de capitales, la persistencia de desequilibrios económicos, el desempleo y la pobreza, entre otros problemas. La solución de estos problemas no era la retirada estatal, como se había pregonado unos años antes, sino la creación de condiciones institucionales que contribuyeran a estabilizar las expectativas de los decisores económicos y permitieran atender a los grupos que quedaban excluidos del mercado, mediante políticas de combate a la pobreza, fortalecimiento de la sociedad civil y desarrollo del capital social.

Este redescubrimiento teórico y político del Estado expresa el reconocimiento de la existencia de tensiones y de oposición a las reformas que requieren de un árbitro capaz de administrarlas y de desarticular potenciales conflictos a través de mecanismos de cooptación, disciplinamiento y represión. En el estilizado lenguaje del fortalecimiento institucional, diversos autores han enfatizado la necesidad de un Estado y de elites estatales fuertes, de una fuerte voluntad política para neutralizar las presiones contrarias a las reformas (SMITH \& KORZENIEWICS, 1997; TORRE, 1997).

Se planteaba además la necesidad de cultivar la paciencia social y apuntalar los mecanismos ideológicos que compensaran la previsible imposibilidad de que las reformas exhibieran un desempeño satisfactorio sostenido (NAÍM, $2000)^{8}$. Desde estas visiones, la necesidad de que el Estado sea impermeable a demandas sociales que no puedan ser satisfechas dentro de los límites de la estrategia reformista y del desarrollo orientado al mercado queda fuera de discusión, como también quedan fuera de discusión los fines del Estado. El debate se centra fundamentalmente en el análisis de las combinaciones adecuadas de recursos que permitan al Estado mejorar su efectividad y eficiencia (LARRETA \& QUINTANA, 1998). De este modo, las estrategias de desarrollo, y especialmente los obstáculos y dilemas del desarrollo en países periféricos, se vuelven cuestiones técnicas más que políticas y las contradicciones propias de las sociedades capitalistas se convierten en problemas de mal uso de recursos y de falta de capacidades institucionales. A la vez, la idea de que instituciones estatales fuertes pueden superar estos obstáculos y contradicciones lleva implícita una noción del Estado como una entidad con ideas e intereses propios, autonomizada de la estructura económica y de las relaciones de poder vigentes (SCHAMIS, 1999) $^{9}$.

El Banco Mundial no sólo adoptó el discurso de las 'capacidades institucionales', sino que también desarrolló parte importante de este aparato conceptual a lo largo de los 90 . En un documento de 1991 se pueden identificar los primeros antecedentes de esta posición. Entonces, el Banco planteaba la necesidad de un Estado que tuviera una perspectiva favorable al mercado (marketfriendly), esto es, que fuera capaz de catalizar las oportunidades económicas, mantener el orden público, invertir en capital humano, construir infraestructura y proteger el medioambiente (Banco Mundial apud VILAS, 1998).

\footnotetext{
8 La crítica de estos argumentos excede los límites de este artículo, pero sería importante reflexionar desde una perspectiva democrática acerca de las enormes implicancias de las ideas de un Estado fuerte y protegido contra presiones sociales.

9 Con este comentario no se quiere sugerir que el Estado está inmediatamente subordinado a algún interés particular, sino que no debería ignorarse que el Estado expresa relaciones sociales históricamente específicas que exceden sus propios límites.
} 
Más claramente, en otro documento publicado en 1992, se señalaba que, con algunas excepciones, los proyectos de ajuste estructural no habían logrado los objetivos esperados debido a problemas de gobernabilidad (v.g. la falta de una clara diferenciación entre las esferas pública y privada, la falta de marcos legales adecuados, el exceso de regulaciones, la arbitraria intervención gubernamental, la incertidumbre e impredictibilidad en la aplicación de las leyes y la falta de procesos transparentes de toma de decisiones). Se explicaba que estos problemas originan corrupción y gasto, limitan la competencia, erosionan la autoridad de los gobiernos y fomentan el incumplimiento de las decisiones y regulaciones, dañando la confianza de los actores económicos y creando un ambiente hostil al desarrollo. Se concluía que los gobiernos deben garantizar la provisión de bienes públicos tales como la formulación de normas y la rectificación de las fallas de mercado. El Estado debe además brindar educación, cuidado de la salud y servicios de infraestructura para los pobres, ya que los mercados difícilmente tengan interés en ofrecer servicios a estos grupos sociales. Pero, como los gobiernos no están naturalmente calificados para ofrecer bienes, es necesario construir capacidades que les permitieran cumplir con estas tareas eficaz y eficientemente (BANCO MUNDIAL, 1992).

En el muy difundido Informe sobre Desarrollo Mundial: El Estado en un Mundo en Transformación de 1997, el Banco Mundial centró su análisis en el rol insustituible del Estado como garante de la ley, la estabilidad económica, la prevención de las fallas de mercado y la protección de los grupos vulnerables y del ambiente. Aquí, el 'Estado deseable' garantiza la disciplina fiscal, se subordina a los tratados económicos internacionales, aplica impuestos al consumo en lugar de otros impuestos considerados distorsivos y, en general, ofrece condiciones atractivas para los inversores privados. El informe también abogaba por la apertura de los sectores de infraestructura y de los servicios sociales a las fuerzas de mercado (BANCO MUNDIAL, 1997) ${ }^{10}$.

Esta brevísima revisión muestra que no estamos ante una mera reversión de las premisas antiestatistas neoliberales, sino más bien ante un

\footnotetext{
10 Una aguda crítica a este informe puede encontrarse en Panitch (1998).
}

decálogo para la adecuación de los estados al escenario mundial de integración económica y movilidad del capital o, como señala Radice, una reestructuración "en torno a un proyecto específicamente capitalista de desarrollo" (RADICE, 2004, p. 14). La intervención del Estado ya no es vista como intrínsecamente indeseable, pero esto no significa que se ponga en cuestión la idea de que el mercado es la instancia preferida de articulación social.

La necesidad que el Estado moldee las relaciones sociales locales en función de los movimientos de capital global se expresa aún más claramente en una publicación editada por el Banco Mundial que revisa las reformas realizadas en América Latina durante los ' 90 y propone políticas para la década siguiente. Aquí, Shahid Burki y Guillermo Perry, vice-Presidente y economista jefe del Banco Mundial para América Latina y el Caribe respectivamente señalan sin rodeos que los estados latinoamericanos deben mediar entre sus sociedades y los movimientos internacionales de capital y proteger a los inversores contra las crisis financieras y monetarias (BURKI \& PERRY, 1998).

\section{LA SEGUNDA ETAPA DE REFORMAS EN ARGENTINA}

En el caso argentino, la preocupación por el fortalecimiento del Estado y el impulso a las reformas de segunda generación fueron precedidos por las dificultades financieras del Estado y por la agudización de los conflictos sociales mencionados más arriba. Luego de la crisis financiera mexicana de 1994, el Banco Mundial reconoció que el tipo de cambio fijo de la Convertibilidad, al limitar el margen de autonomía de las políticas monetarias del Banco Central, hacía al país especialmente vulnerable a las crisis financieras internacionales (BANCO MUNDIAL, 1996a). Sin embargo, la estrategia de asistencia de la institución para el año 1995 no proponía revisar los fundamentos de este régimen sino 'profundizar el proceso de ajuste' manteniendo las políticas macroeconómicas, implementando la reforma de los estados provinciales y la reestructuración del sistema financiero. Más concretamente, el Banco recomendaba una reestructuración del sistema bancario y un desarrollo del mercado de capitales que mejorara la eficiencia de los mercados financieros y redujera el costo del crédito (idem, p. 37). Planteaba además la creación de un Fondo Fiduciario de Capitalización Bancaria que sirviera 
para proteger a los bancos en caso de corridas especulativas contra el peso, la absorción de los bancos más pequeños por parte de instituciones más fuertes para reducir el riesgo bancario, la privatización de los bancos públicos provinciales para evitar que el Estado nacional tuviera que hacer aportes presupuestarios a los mismos y la del Banco Nación. Siguiendo estas recomendaciones, se creó un fondo que durante los años siguientes sería utilizado asiduamente para asistir a los bancos. Además, con el fin de obtener asistencia financiera del Banco Mundial, varias provincias transfirieron sus bancos públicos al sector privado. En cambio, la privatización del Banco de la Nación Argentina, principal banco público del país de propiedad del Estado nacional, no se concretó debido a la fuerte oposición política que tuvo esta propuesta.

El Banco Mundial proponía además la reestructuración de los mercados de trabajo - a cuya rigidez atribuía tanto la falta de competitividad de la economía argentina como las altas tasas de desempleo -, la creación de una red de seguridad social, la reforma del sistema de salud, la racionalización de la educación superior, la reforma del sistema judicial, la implementación de esquemas de promoción de exportaciones, la privatización de los aeropuertos, la formulación de programas para el desarrollo de la agricultura y minería, la implementación de proyectos de desarrollo de transportes, protección contra inundaciones, provisión de agua y contaminación (idem).

Aunque sólo algunas de estas propuestas se tradujeron en proyectos de asistencia concretos que se fueron implementando en función de prioridades coyunturales y de presiones diversas y contradictorias sobre el Estado, la ampliación de las preocupaciones del Banco Mundial estuvo acompañada por una multiplicación de préstamos para el desarrollo de proyectos. Así, el Banco asumió un rol protagónico en la formulación e implementación de programas en áreas de intervención estatal ligadas con la reproducción social. Esto contrasta tanto con el rol que la institución había tenido desde su creación en el financiamiento de proyectos de desarrollo, como con los programas de ajuste estructural tendientes a alcanzar equilibrios macroeconómicos que el Banco había patrocinado internacionalmente durante los '80 y principios de los '90.
Sin embargo, como ya se sugirió, las nuevas preocupaciones no constituyeron una ruptura con las perspectivas previas sino el reconocimiento de un conjunto más amplio de necesidades relacionadas con la continuidad del ajuste estructural. Proyectos destinados a fines tan diversos como asistir a los desempleados, brindar apoyo alimentario a grupos considerados vulnerables, mejorar la calidad de la enseñanza media y universitaria, reestructurar los sistemas de salud y de seguridad social planteaban explícitamente que las reformas económicas (apertura económica, reducción de costos para el capital, privatización etc.) eran la clave del crecimiento económico y de la inclusión social en el mediano plazo, más allá de sus costos inmediatos. Hasta tanto se lograran estos objetivos, los préstamos de la institución debían servir para aliviar los impactos políticamente disruptivos del ajuste y para reorganizar las áreas del Estado a cargo de las políticas sociales, en línea con los preceptos neoliberales de privatización y/o arancelamiento de los servicios destinados a los grupos de mayor poder adquisitivo y de oferta de servicios mínimos a grupos focalizados considerados vulnerables. También planteaban que la excesiva intervención estatal y las deficiencias de los propios trabajadores y desempleados eran los factores que obstaculizan su inserción en los mercados de trabajo, lo cual hacía necesario desregular estos mercados, eliminar un conjunto de derechos de los trabajadores para reducir el costo de la fuerza de trabajo y capacitar a los trabajadores para que se adaptaran a las demandas de los empleadores. En el mismo sentido, se proponía reformar el sistema educativo para adecuar la educación a las necesidades y demandas del mercado. Para sortear la coyuntura de crisis, se debía priorizar la creación de redes de asistencia a grupos sociales ‘vulnerables’ (básicamente provisión de algunos alimentos y controles básicos de salud para madres y niños pequeños). Paralelamente se alentaba la eliminación la gratuidad y universalidad de los servicios de salud y educación, con el argumento de que era necesario evitar que sectores de ingresos medios usaran los servicios y desviaran parte de los escasos recursos disponibles, limitando la posibilidad de brindar atención a quienes realmente la necesitaban ${ }^{11}$. A la vez, se alentaba la

11 Aunque el avance en el arancelamiento de los servicios ha sido dispar, el deterioro de los mismos dio lugar a una 
incursión de capital privado en los sistemas públicos de salud, seguridad social y educación. ${ }^{12}$

Estas nuevas políticas fueron acompañadas por apelaciones a la participación de la sociedad civil, la cual era considerada una herramienta administrativa que facilitaba la implementación de las políticas, a la vez que creaba un sentido de propiedad entre los beneficiarios de las políticas (BANCO MUNDIAL, 1997). En esta línea, el Banco alentó formas de autoayuda y condicionó el otorgamiento de algunos préstamos destinados a programas de asistencia social a la participación de los beneficiarios de estos programas.

A partir de 1999, el Banco Mundial implementó un esquema de diálogo destinado a debatir la Estrategia de Asistencia al País que guiaría su intervención durante los siguientes cinco años (Country Assistance Strategy - CAS) al que convocó a los diversos niveles de gobierno y a organizaciones de la sociedad civil (BANCO MUNDIAL, 2000). Entre 1999 y 2000 se desarrollaron diversas reuniones en las que se debatieron las propuestas del Banco Mundial, pero se dejaron fuera de discusión las premisas económicas de disciplina fiscal, desregulación, subsidiariedad de la intervención del estado y políticas sociales focalizadas (KARAMAN, TRINCHERO \& WOODS, 2001). Por otra parte, el Banco no asumía ningún compromiso de tomar en cuenta las conclusiones y recomendaciones que surgieran de estas reuniones. A pesar de estos límites, la convocatoria a la participación sirvió para revestir de consenso social a los programas de reforma.

La contracara de estas nuevas preocupaciones fue la transformación de las modalidades de formulación, implementación y evaluación de políticas estatales, de las formas de asignación de recursos públicos y de las relaciones laborales al

gradual privatización de hecho, ya que los grupos de mayor poder adquisitivo han optado por pagar por obtener servicios de mayor calidad, mientras que el resto de la población ha seguido dependiendo de la provisión estatal de servicios cada vez más deteriorados por la falta de recursos.

12 En este párrafo se sintetizan muy brevemente las explicaciones y los objetivos formales de un número considerable de documentos de proyectos de reformas sectoriales auspiciados por el Banco Mundial durante la década del '90 en la Argentina. Pueden consultarse, entre otros, Banco Mundial (1995, 1996a y 1996a); Experton (1999); Argentina (2000). interior del estado. En el contexto de creciente escasez fiscal que afectó al estado argentino desde mediados de los '90, el financiamiento del Banco permitió a diversas agencias estatales poner en marcha programas, contratar personal, ganar visibilidad política y crear redes clientelares políticas y profesionales. A partir de esta dinámica, se reformularon los objetivos de estas agencias, la asignación de recursos, la configuración de sus plantas de personal, los instrumentos administrativos y las relaciones jerárquicas al interior de la administración pública. Las agencias obtenían recursos siempre y cuando estuvieran dispuestas a adoptar los objetivos estratégicos y a llevar adelante las reformas institucionales sugeridas por el Banco. Como además sus préstamos sólo cubrían una parte de los costos de la formulación e implementación de los programas, el estado debía derivar parte de sus propios recursos a la implementación de estos proyectos. Para la gestión de proyectos, se exigía la conformación de unidades ejecutivas y la contratación de consultores, supuestamente más eficientes y eficaces que la burocracia permanente, y la adopción de los sistemas de control y auditoría del Banco Mundial, que se superponían con los controles propios de la burocracia.

De este modo, aunque en la mayoría de los casos los programas no cumplieron con sus objetivos formales (reducir la pobreza y el desempleo, mejorar el acceso a la educación y la salud, entre otros), los mismos ampliaron el espacio de participación del Banco en la formulación e implementación de políticas y fueron el vehículo por el cual sus concepciones acerca de las instituciones estatales, de los problemas sociales y de las soluciones se convirtieron en referencias obligadas de la acción de las agencias estatales. En otros términos, la preocupación por mejorar las capacidades institucionales del estado fue la llave que permitió que el Banco Mundial se involucrara directamente en la gestión de un amplio conjunto de áreas estatales o, lo que es lo mismo, que la acción del estado en su conjunto (y no sólo las políticas macroeconómicas) quedara bajo su escrutinio.

Los esfuerzos de fortalecimiento institucional tuvieron corta vida. Desde 1999, en el contexto de empeoramiento de la situación económica y financiera del país, se fue reduciendo el monto de los préstamos del Banco Mundial, tanto para evitar el engrosamiento de la deuda pública, como 
para ahorrar los recursos que el estado debía aportar para la implementación de los programas. Consecuentemente, se eliminaron numerosos programas y se limitó significativamente el alcance de otros.

\section{EL ‘MEJOR’ ESTADO ES EL QUE MENOS GASTA}

Los últimos años de la década de los '90 se caracterizaron por la caída de la recaudación impositiva y las elevadísimas tasas de interés que el Estado debía pagar para refinanciar su abultada deuda pública. Las dificultades fiscales del Estado se enmarcaban en un paisaje recesivo, de caída de las inversiones, reducción de salarios y niveles de desempleo y pobreza inéditos (KATZ, 2001).

En este escenario no sólo se limitaron los programas iniciados unos años antes sino que también se desplazaron los ejes centrales de la acción del Banco Mundial. Aunque la institución no abandonó formalmente sus argumentos acerca del fortalecimiento del Estado y la protección social, ya en 1997 la asistencia había comenzado a centrarse en la frágil situación macroeconómica. En sus diagnósticos y recomendaciones, la institución señalaba que el Estado argentino debía garantizar el valor de la moneda y proteger al sistema financiero contra las corridas especulativas como un modo de "enviar fuertes señales a los mercados acerca de su credibilidad en el largo plazo” (BANCO MUNDIAL, 1998, p. 2).

En 1998, luego de que el país alcanzara un acuerdo con el FMI por el cual este último otorgaría una préstamo ‘precautorio’ que podría utilizarse en caso de que las consecuencias de la crisis asiática lo hicieran necesario, el Banco Mundial aprobó un préstamo contingente (Special Repurchase Facility Support-REPO) y un Préstamo Especial de Ajuste Estructural (Special Structural Adjustment Loan - SSAL) que tenían por objeto mitigar las consecuencias de la crisis financiera internacional iniciada en el sudeste asiático en 1997. Los acuerdos con el FMI y el Banco Mundial comprometían al Estado argentino a mantener redes de protección social y a avanzar en las reformas de los sistemas de educación y salud, profundizar el ajuste fiscal, reestructurar el sistema bancario, privatizar el Banco de la Nación, otorgar inmunidad legal a los directores del Banco Central, avanzar en la flexibilización de las relacio-nes laborales con el fin de reducir el costo del trabajo, abrir el sistema pú- blico de salud a la participación de actores privados, racionalizar los programas de asistencia social y reducir el déficit de los estados provinciales ${ }^{13}$. Estas medidas eran condiciones para otorgar fondos que le permitirían al Banco Central asistir a los bancos privados amenazados por movimientos especulativos y que cubrirían las necesidades de financiamiento del estado durante 1999 (idem; BANCO MUNDIAL, 1999b).

En 1999, el Banco Mundial otorgó una garantía a los bonos que el Estado argentino ofrecería en los mercados financieros internacionales, con la expectativa de que esta garantía contrarrestara la desconfianza de los inversores internacionales (BANCO MUNDIAL, 1999b).

Como se señaló más arriba, en el contexto del agravamiento de la crisis fiscal y de inestabilidad financiera internacional, el énfasis de la asistencia del Banco Mundial viró de la protección social y el fortalecimiento institucional del Estado a los problemas macroeconómicos y fiscales, la volatilidad de los mercados financieros y el financiamiento de la deuda pública. Es interesante señalar que, a diferencia de lo que había sucedido en los años previos, en los que la falta de cumplimiento de los compromisos asociados a los préstamos por parte del Estado argentino se había resuelto mediante negociaciones con el Banco y el FMI, en esta etapa los desembolsos se interrumpieron cada vez que Argentina no cumplió con sus obligaciones. Para reestablecer el flujo de fondos, estas instituciones demandaron sucesivos ajustes fiscales que no harían sino agravar la recesión y los desequilibrios.

\section{LA PROFUNDIZACIÓN DEL AJUSTE Y EL ESTALLIDO DE LA CRISIS}

En 1999, el candidato peronista Eduardo Duhalde sufrió una derrota electoral frente a la opositora Alianza para el Trabajo, la Justicia y la Educación liderada por Fernando de la Rúa. A fin de ese año, de la Rúa asumió la Presidencia del país. Uno de los ejes de su campaña electoral había

\footnotetext{
13 Como el Estado nacional no contaba con instrumentos legales para imponer ajustes sobre las administraciones provinciales según lo demandaba el FMI, el Banco Mundial y el Banco Interamericano de Desarrollo ofrecieron créditos a las provincias que aceptaran realizar estos ajustes. Así, con el incentivo de obtener recursos adicionales, varios gobiernos provinciales se comprometieron a llevar adelante programas de reducción de gastos exigidos por el Ministerio
} de Economía nacional. 
sido la crítica al desmantelamiento del aparato del estado de los años previos y a sus consecuencias económicas y sociales. Tan pronto como asumió el nuevo gobierno, el Banco Mundial presentó un plan que, con el fin de reducir el gasto público, proponía reducir el personal del Estado, cerrar escuelas, limitar el número de maestros y de personal de salud, arancelar y privatizar algunos servicios y reformar el sistema judicial.

Ante el crecimiento acelerado del riesgo país, la suba de las tasas de interés y la salida de capitales que signaron la gestión de de la Rúa, el gobierno hacía propias las exigencias de ajuste cada vez más estrictas de las instituciones financieras internacionales. Así, en abril de 2000, se puso en marcha un ajuste fiscal que incluyó la reducción de salarios nominales de parte de los empleados públicos, el aumento de impuestos sobre el consumo y la eliminación de varias agencias estatales. El recorte de gastos alcanzó también a varios de los proyectos de asistencia social auspiciados por el propio Banco Mundial, contradiciendo la premisa de que los programas sociales debían acudir en ayuda de grupos vulnerables en momentos de crisis ${ }^{14}$.

El ajuste fiscal no fue suficiente para que el Estado recobrara la confianza de los inversores y lograra refinanciar su deuda a tasas de interés más bajas (DAMILL, FRENKEL \& MAURIZIO, 2002). Sin embargo, fue suficiente para generar crecientes cuestionamientos políticos y fogonear el conflicto social. Provocó además una caída del consumo que restringió aún más los ingresos estatales. Frente a lo que se definió como una 'crisis de confianza', a fines del 2000, el gobierno anunció la obtención de un 'blindaje financiero', constituido a partir de créditos del FMI, el Banco Mundial, el Banco Interamericano de Desarrollo, el Estado español y algunos bancos privados internacionales que podría usarse para cumplir con los compromisos derivados del endeudamiento público (Argentina, 2001). El anuncio de este crédito tuvo como contrapartida el compromiso de llevar adelante un nuevo ajuste fiscal y de reestructurar los sistemas de salud y de jubilaciones, con el fin de reducir el aporte estatal y generar condiciones para el ingreso de capital pri-

14 Acerca de la importancia de la contención social, ver Banco Mundial (2000). vado. Este 'blindaje' fue presentado como un instrumento que reduciría las tasas de interés que el país tendría que pagar para refinanciar su deuda y que contribuiría a la reactivación de la economía. De hecho, era un mecanismo de protección de los acreedores frente al riesgo de cesación de pagos por parte del Estado.

El 'blindaje’ tampoco logró aliviar la crítica situación fiscal y financiera y el incumplimiento de las metas de ajuste acordadas con el FMI complicó las negociaciones con la institución. Luego de que las autoridades económicas anunciaran su intención de cambiar bonos de corto plazo por otros de mayor plazo y mayores tasas de interés, se reanudaron las negociaciones con el Fondo, las cuales resultaron en un nuevo compromiso de garantizar la paridad cambiaria, eliminar el déficit fiscal, respetar la autonomía del Banco Central y profundizar la apertura comercial.

Una vez más, el compromiso de ajuste no sirvió para mejorar la situación financiera pero agravó la recesión y el descontento social. La conjunción de desconfianza por parte de los acreedores, la aceleración de la fuga de capitales y el estallido del descontento social culminaron en la renuncia del Presidente De la Rúa a fines del 2001, la cesación del pago de la deuda pública y la devaluación de la moneda, tras once años de tasa de cambio fija.

Durante la última etapa de vigencia del Plan de Convertibilidad, la volatilidad financiera creó oportunidades extraordinarias de ganancias para capitales financieros, mientras que la profundización del ajuste intensificó la subordinación del Estado y de sus políticas a los requerimientos de las instituciones financieras internacionales. Los esfuerzos del gobierno por responder a estos requerimientos implicaron que el Estado asumiera renovados compromisos de reforma. El agravamiento de la crisis no llevó al Banco Mundial a revisar su adhesión a la estrategia de reformas llevadas adelante durante la década y al mantenimiento del Plan de Convertibilidad, sino a abandonar su protagonismo en el proceso de reformas y a seguir el liderazgo del FMI en la nueva etapa de profundización del ajuste fiscal ortodoxo ${ }^{15}$.

15 Según se desprende de los dichos de un funcionario de la Misión del Banco Mundial en la Argentina (entrevista personal). También declaraciones de Myrna Alexander, directora de la Misión del Banco Mundial en la Argentina, en el diario Página 12 de 24 de abril de 2002. 
La devaluación de la moneda, que a principios de 2002 puso fin a una década de paridad entre el dólar estadounidense y el peso argentino, fue seguida por un agravamiento de la recesión y la inestabilidad macroeconómica y financiera que sólo comenzaron a revertirse cuando el gobierno provisorio encabezado por el entonces senador peronista Eduardo Duhalde comenzó a apartarse de los requerimientos del FMI y estableció algunos controles y mecanismos de regulación de los mercados financieros, mientras negociaba las nuevas reglas de juego post devaluación con diversos actores económicos. Estos controles, sumados al alivio financiero que siguió a la suspensión del pago de la deuda pública y a las ventajas competitivas derivadas de la devaluación, lograron estabilizar la economía, crear cierta holgura fiscal y poner fin al largo período de estancamiento iniciado en 1998.

En 2003 se llevaron a cabo elecciones que llevaron a la Presidencia a Néstor Kirchner, el candidato oficialista que compitió con aspirantes provenientes de otras corrientes del peronismo, del radicalismo, de fuerzas de izquierda y de otros partidos menores. En el contexto de relativa mejora de la economía, el nuevo gobierno amplió su espacio de negociación de las condiciones de la integración económica global del país, esto es, llevó adelante una reestructuración de la deuda pública que supuso un recorte importante del capital a pagar a los acreedores y comenzó a negociar más que aceptar sin discutir - los requerimientos de ajuste ortodoxo del FMI'16.

\section{CONCLUSÕES}

Las reformas llevadas a cabo en Argentina durante los '90 están lejos de haber resultado en un Estado capaz de responder más eficazmente a las demandas sociales y de generar condiciones para un desarrollo sustentable. Pero el énfasis en cuestiones sociales y estructurales ha sido una forma de subordinar el conjunto de la acción del Estado a los imperativos de la acumulación de capital y de integración con la economía global, con lo cual no puede decirse que las reformas hayan fracasado $^{17}$.

16 Es necesario señalar que la política económica del gobierno del Presidente Kirchner no se agota en estas medidas pero su análisis excede los límites de este trabajo.

17 Para una discusión teórica de este tema, ver Cammack (2001).
En este proceso, las instituciones financieras internacionales han actuado como representantes políticas de los intereses del capital global, exigiendo un tipo específico de reestructuración del estado ${ }^{18}$ que se concretaría mediante reformas en la legislación del país, en la configuración del aparato estatal y en la orientación de las políticas estatales. Su capacidad de influencia se potenció, independientemente de los montos de sus préstamos, porque su respaldo a las reformas fue una señal positiva para los mercados internacionales. Por otra parte, aunque estos préstamos fueran una mínima porción del flujo de capitales hacia el país, la posibilidad de obtener recursos adicionales en un contexto de escasez fiscal fue significativa para las agencias estatales que se volcaron masivamente a desarrollar programas que, por sus objetivos y orientaciones, cumplían con los requisitos del Banco Mundial para otorgar financiamiento. De este modo, la dependencia respecto de los recursos se transformó en una dependencia respecto de la producción ideológica del Banco ${ }^{19}$. En la medida en que el Estado argentino aceptó el diagnóstico que identificaba a la mala asignación de recursos públicos, las políticas equivocadas y los problemas de gestión de las instituciones estatales como los principales obstáculos para el desarrollo, la forma de integración económica global del país y sus consecuencias quedaron fuera de discusión (CASABURI \& TUSSIE, 2000).

Tanto la última etapa de crisis como la recuperación económica se enmarcaron en un nuevo consenso político y social crítico del neoliberalismo y de las reformas llevadas a cabo durante los '90. Nada de esto significa, sin embargo, que se hayan revertido las transformaciones estructurales de la economía ni la reestructuración del aparato del Estado ocurridas durante los '90. En este sentido es que puede decirse que las reformas han excedido ampliamente los aspectos funcionales e institucionales para transformar a la sociedad y al Estado de manera profunda y duradera.

18 Sobre las características de la reestructuración de los estados requeridas por el Banco Mundial, ver Panitch (1998).

19 Sobre la relación entre asistencia financiera y la aceptación de las perspectivas ideológicas del Banco Mundial, ver Wade (2001). 
Ruth Felder (felder_ruth@yahoo.com.ar) é Mestre em Ciência Política pela Universidade de York, Canadá e Professora da Faculdade de Ciencias Sociales da Universidade de Buenos Aires, Argentina.

\section{REFERÊNCIAS BIBLIOGRÁFICAS}

BASUALDO, E. 1999. Acerca de la naturaleza de la deuda externa y la definición de una estrategia política. Buenos Aires : La Página.

BONNET, A. 2003. El comando del capital-dinero y las crisis latinoamericanas. In : BONEFELD, W. \& TISCHLER, S. A 100 años del ¿Qué hacer? Leninismo, crítica marxista y la cuestión de la revolución hoy. Buenos Aires : Herramienta.

BURKI, S. \& PERRY, G. 1998. Beyond the Washington Consensus. Institutions Matter. Washington : World Bank.

BURNHAM, J. 1999. The IMF and the World Bank: Time to Merge. The Washington Quarterly, Washington, v. 22, n. 2, p. 101113, Spring.

CAMMACK, P. 2002. The Mother of All Governments: The World Bank's Matrix for Global Governance. In : HUGHES, S. \& WILKINSON, R. Global Governance: Critical Perspectives. London : Routledge.

CASABURI, G. \& TUSSIE, D. 2000. La sociedad civil y los Bancos Multilaterales de Desarrollo. In : TUSSIE, D. Luces y sombras de una nueva relación: El Banco Interamericano de Desarrollo, el Banco Mundial y la Sociedad Civil. Buenos Aires : Temas.

DAMILL, M.; FRENKEL, R. \& MAURIZIO, R. 2002. Argentina: Una década de Convertibilidad. Un análisis del crecimiento, el empleo y la distribución del ingreso. Santiago : Organización Internacional del Trabajo.

EXPERTON, W.; BECERRA, M.; CESILINI, S.; GUTIÉRREZ, S.; IZQUIERDO, R.; PUECH, R.; ROTEN, D. \& VEGAS, E. 1999. Desafíos para la Nueva Etapa de la Reforma Educativa en Argentina. LCSHD Paper Series, World Bank, Washington, D. C., n. 46, Sep.

GAMBINA, J. 2003. Sobre la deuda externa pública de la Argentina: ¿qué hace el gobierno y qué debiera hacerse? Buenos Aires : Fundación de Investigaciones Sociales y Políticas.

KARAMAN, G.; TRINCHERO, H. \& WOODS,
M. 2001. La consulta del Banco Mundial a la sociedad civil en la Argentina: Economía política de la participación. Realidad Económica, Buenos Aires, n. 180, p. 91-111, jul.-ago.

KATZ, C. 2001. La crisis económica argentina: interpretaciones y propuestas. Trabalho apresentado no Encuentro de Economistas de Izquierda, Buenos Aires. Disponível em : <http:/ $/ \mathrm{w} w \mathrm{w}$. g e o c i t i e s. c o m/ e conomistas_de_izquierda/ claudiokatzlacrisis.htm>. Acesso em : 29.abr.2009.

KULFAS, M. \& SCHORR, M. 2003. Deuda externa y valorización financiera en la Argentina actual. Factores explicativos del crecimiento del endeudamiento externo y perspectivas ante el proceso de renegociación”. Realidad Económica, Buenos Aires, n. 198, ago.-sep.

LARRETA, H. R. \& QUINTANA, M. 1998. Domando al elefante blanco: pasos para construir un estado que funcione. Buenos Aires : Temas.

MOSLEY, P.; HARRIGAN, J. \& TOYE, J. 1991. Aid and Power: The World Bank \& PolicyBased Lending. London-New York : Routledge.

NAÍM, M. 2000. Fads and Fashion in Economic Reforms: Washington Consensus or Washington Confusion? Third World Quarterly, v. 21, n. 3, p. 505-528, Jun. Disponível em : <http:/ /www.imf.org/external/pubs/ft/seminar/1999/ reforms/Naim.HTM>. Acesso em : 28.abr.2009.

PANITCH, L. 1998. The State in a Changing World: Social-Democratizing Global Capitalism? Monthly Review, New York, v. 50, n. 5, p. 11-23, Fall.

RADICE, H. 2004. Taking Globalization Seriously. In : PANITCH, L.; LEYS, C.; ZUEGUE, A. \& KONINGS, M. The Globalization Decade: A Critical Reader. London : Merlin Press.

ROXBOROUGH, I. 1997. Citizenship and Social Movements Under Neoliberalism. In : SMITH, W. \& KORZENIEWICZ, R. Politics, 
Social Change and Economic Restructuring in Latin America. Boulder : North-South CenterLynne Reinner.

SCHAMIS, H. 1999. Distributional Coalitions and the Politics of Economic Reform in Latin America. World Politics, Baltimore, v. 51, n. 2, p. 236-268, Jan.

SCHVARTZER, J. 1997. Ajuste, reestructuración, políticas industrials y globalización económica. In : ASAMBLEA GENERAL DE CLACSO, 18, Seminário : Balance y perspectivas de las ciencias sociales en américa latina y el Caribe, Buenos Aires. Mimeo. Buenos Aires : Clacso, nov.

SMITH, W. \& KORZENIEWICZ, R. 1997.

Latin America and the Second Great Transformation. In : SMITH, W. \& KORZENIEWICZ, R. Politics, Social Change and Economic Restructuring in Latin America. Boulder : North-South Center-Lynne Reinner.

TEICHMAN, J. 1997. Mexico and Argentina: Economic Reform and Technocratic DecisionMaking. Studies in Comparative International
Development, New Brunswick, v. 32, n. 1 p. 31-55, Spring.

TORRE, J.C. 1997. The Politics of Transformation in Historical Perspective. In : SMITH, W. \& KORZENIEWICZ, R. Politics, Social Change and Economic Restructuring in Latin America. Boulder : North-South Center-Lynne Reinner.

TUSSIE, D. \& BOTZMAN, M. 1990. Sweet Entanglement: Argentina and the World Bank 1985-9. Development Policy Review, London, v. 8, n. 4, p. 393-409, Dec.

VILAS, C. 1998. Más allá del 'Consenso de Washington’? Un enfoque desde la política de algunas propuestas del Banco Mundial sobre reforma institucional. Reforma y Democracia, Caracas, n. 18, p. 25-76. Disponível em : <http:/ /campus.usal.es/ iberoame/americalatinahoy/ A L H - P D F - T I F F / A L H v o 126 / ALHvol26vilas.pdf>. Acesso em : 28.abr.2009.

WADE, R. 2001. Showdown at the World Bank. New Left Review, London, n. 7, p. 124-137, Jan.-Feb.

\section{OUTRAS FONTES}

ARGENTINA. 2000. Guía de Programas sociales del Ministerio de Desarrollo Social y Medio Ambiente. Sistema de Información, Monitoreo y Evaluación de Programas Sociales (Siempro). Buenos Aires : Ministerio de Desarrollo Social y Medio Ambiente.

ARGENTINA. Secretaria de Finanças. 2001. Cuenta de inversión 2001. Anexo K Acontecimientos financieros relevantes del período. Buenos Aires : Ministerio de Economía, Obras y Servicios Públicos. Disponível em : <http://www.mecon.gov.ar/hacienda/cgn/ cuenta/2001/sdp/anexok.htm>. Acesso em 28.abr.2009.

BANCO MUNDIAL. 1992. Governance and Development. Washington : World Bank.

BANCO MUNDIAL. 1995. Argentina. Higher Education Reform Project. Washington : World Bank.

BANCO MUNDIAL. 1996a. Argentina Country Assistance Review. Washington : World Bank.
BANCO MUNDIAL. 1996b. Argentina. The Convertibility Plan: Assessment and Potential Prospects. Washington : World Bank.

BANCO MUNDIAL. 1997. El Estado en un mundo en transformación: informe sobre el desarrollo mundial 1997. Washington : World Bank.

BANCO MUNDIAL. 1998. Argentina Special Repurchase Facility Support Loan (REPO). Washington : World Bank.

BANCO MUNDIAL. 1999a. Argentina. Health Insurance for the Poor. Washington : World Bank.

BANCO MUNDIAL. 1999b. Argentina Policy Based Guarantee Operation. Washington : World Bank.

BANCO MUNDIAL. 2000. Memorandum of the President of the International Bank for Reconstruction and Development to the Executive Directors on a Country Assistance Strategy of the World Bank Group for the Argentine Republic. Washington : World Bank. 
INSTITUTIONAL REFORM AND GLOBAL INTEGRATION: WORLD BANK INTERVENTION IN ARGENTINA DURING THE 1990s

\section{Ruth Felder}

The process of economic and institutional reform that was carried out in Argentina over the decade of the nineties led to deep transformations in the country's economic structure, State forms, action and configurations of its institutional apparatus. In this paper I take a look at World Bank intervention in the different stages of this process, paying particular attention to the role that this institution played in the early state of reduction of the size of the State apparatus and opening up to the market. I then go on to analyze arguments and projects regarding the strengthening of State institutional capacities and the creation of networks of social containment developed within the framework of what has been referred to as "Second reform of the State" in the mid-nineties. Lastly, I will concentrate on how these priorities related to strengthening the State were abandoned and how projects related to fiscal adjustment and public debt management acquired greater importance within the context of macro-economic imbalances and the recession that characterized the latter part of the nineties. Through analysis of the historical dynamics of World Bank intervention in the reform process, I will argue that, beyond the formal goals of numerous World Bank projects, the institution has had a mediating role between the requirements of global economic integration and the Argentine State, in both the shrinking and strengthening phases of State intervention. Projects have been oriented toward transforming these requirements into concrete institutional frameworks and state policies that increased the country's integration into the global economy. These priorities have been manifested through the crisis of the late 1990s and the beginning of the current decade.

KEYWORDS: Argentina; World Bank; State reform; neo-liberalism. 


\section{Ruth Felder}

Le processus de réformes économiques et institutionnelles mis en oeuvre en Argentine dans les années 1990 a profondément transformé la structure économique du pays, les formes d'action de l'État et la configuration de son appareil institutionnel. Dans ce travail, je reverrai l'intervention de la Banque Mondiale dans les différentes étapes de ce processus, en soulignant le rôle qu'elle a joué lors de la première étape de réduction de la taille de l'appareil de l’État et de l'ouverture au marché. Ensuite j'étudierai dans les arguments et projets concernés le renforcement des capacités institutionnelles de l'État et la création des réseaux de limitation sociale mis en place dans le cadre de la « Seconde Réforme de l'État » au milieu des années 90. Enfin, j’examinerai non seulement l'abandon des priorités liées au renforcement de l'État, mais aussi le rôle de plus en plus important qu'ont joué les projets liés à l'ajustement fiscal et au contrôle de la dette publique dans le contexte des déséquilibres macroéconomiques et de la récession de la fin des années 1990. À partir de l'analyse du dynamisme historique de l'intervention de la Banque Mondiale dans le processus de réformes, je prônerai qu'au-delà des objectifs formels des nombreux projets de la Banque Mondiale, l'institution a joué un rôle de médiateur entre les demandes d'intégration à l'économie mondiale et l'État argentin, aussi bien dans l'étape de « réduction » que dans celle " d'amélioration » de l'intervention de l'État. Ses projets se sont orientés vers la transformation des demandes en schémas institutionnels et en politiques de l'état concrètes qui ont renforcé l'intégration du pays dans l'économie mondiale. Ces priorités se sont dévoilées lors de la crise de la fin des années 1990 et au début des années 2000.

MOTS-CLÉS : Argentine ; Banque Mondiale ; réforme de l’État ; néo-libéralisme. 